\title{
Investigation of the structure of anti-human seminal plasma protein single-chain antibody and its association with linker peptide length
}

\author{
XIN JIANG ${ }^{1,2}$, JUN ZHAI $^{3}$, DONGKUI SONG ${ }^{1}$, QINGSHAN QU ${ }^{2},{\text { MING } \text { LI }^{2}, \text { LI XING }^{2} \text { and SHUZHAI MIAO }}^{2}$ \\ ${ }^{1}$ Department of Urology, First Affiliated Hospital of Zhengzhou University, Zhengzhou, Henan 450052; \\ ${ }^{2}$ Department of Kidney Transplantation, People's Hospital of Zhengzhou, Zhengzhou, Henan 450003; \\ ${ }^{3}$ Reproductive Center, First Affiliated Hospital of Zhengzhou University, Zhengzhou, Henan 450052, P.R. China
}

Received July 16, 2014; Accepted May 8, 2015

DOI: $10.3892 / \mathrm{mmr} .2015 .3980$

\begin{abstract}
To enhance the activity of seminoprotein single-chain variable fragment $(\gamma-\mathrm{Sm}-\mathrm{ScF})$ antibodies, modulation of the length of the linker peptide, which connects the variable region of the heavy chain $(\mathrm{VH})$ and the light chain (VL) of single-chain antibodies, was performed in the present study. Homologous modeling of single $\mathrm{VH}$ and $\mathrm{VL}$ were performed, respectively. Subsequently, modeling of the whole ScFv sequence, which was previously modified with added linkers of different lengths was also performed, and the $\left(\mathrm{Gly}_{4} \mathrm{Ser}\right)_{\mathrm{n}}$ peptide chain structure was used as the linker. The similarities between $\mathrm{VH}$ and $\mathrm{VL}$ prior to and following the addition of the linker were compared by applying the algorithm of protein similarity, based on spherical coordinates layering. In addition, changes in the fore and aft distance, and diffusion radius were calculated using a MATLAB tool, based on which changes in structural stability were analyzed. Finally, the single-chain antibody was assessed in a nude mouse model. When $n=3$ or $\mathrm{n}=6$, the similarity between the original distance and $\mathrm{VH}$ and VL were the highest, and the fore and aft distance and diffusion radius were relatively close. In addition, the nude mouse model indicated that, when $n=3$ or $n=6$, the inhibitory rate of the single-chain antibody against tumor cells was significantly higher, compared with the other linker peptides of different lengths. The effect of structural changes of the linker peptides in the single-chain antibodies on the whole antibody molecule was examined at different levels using a combination of mathematical modeling, bioinformatics methods and biological experiments. The findings of the present study may provide a foundation for further investigation into the preparation of single-chain antibodies.
\end{abstract}

Correspondence to: Professor Dongkui Song, Department of Urology, First Affiliated Hospital of Zhengzhou University, 1 East of Jianshe Road, Zhengzhou, Henan 450052, P.R. China

E-mail: dongkuisong126@126.com

Key words: seminoprotein, single-chain antibody, linker, diffusion radius, fore and aft distance

\section{Introduction}

Prostate cancer refers to epitheliogenic malignant tumors of the prostate. What is usually termed prostate cancer refers to adenocarcinoma of the prostate. Since the mid to late 1980s, the morbidity and mortality rates of prostate cancer in certain developed areas in China have continued to increase (1). Previous studies have indicated that seminal plasma protein is one of the specific markers of prostate cancer (2). Hao and Liang (3) used 131I to mark the anti-human seminal plasma protein single-chain antibody $(\gamma-\mathrm{Sm}-\mathrm{McAb})$, which was used for the radioimmunoimaging and treatment of prostate cancer. This method was confirmed to be of high sensitivity and specificity, and of beneficial therapeutic effect (3). However, the monoclonal antibodies used originated from murine animals, the repeated use of which may generate anti-mouse antibodies, affecting the therapeutic effect and resulting in allergic reactions (4). Due to the small molecular size, low antigenicity and advantages over other parental antibodies when used within the body, a single chain variable fragment (ScFv) can be used to effectively avoid deficiencies in monoclonal antibodies and has received increasing attention (5). A ScFv is composed of a heavy chain $(\mathrm{VH})$ and a light chain (VL) through linkers of several amino acids (6) (Fig .1). VH and VL are the minimum functional fragments in the binding site of antibodies to antigens, while the linker peptide is used predominantly for connection between the two for fusion expression. (Gly4Ser)n is usually selected as the linker peptide in $\mathrm{ScFv}$ (7), however, the effect of the of the selected n-value on the functional expression of the two variable regions in $\mathrm{ScFv}$ is key to investigating the optimal construct of ScFv. In the present study, a similarity algorithm of spherical coordinates for layered proteins was used. Comparison of changes in the structural models of the changeable structures in single-chain antibodies under different values of $n$, enable the optimal linker length to be determined for maintaining active seminal plasma protein single-antibodies. The results of the present study aim to provide a foundation for the preparation of a single-chain antibody, which may be effective in the treatment of prostate cancer. 


\section{Materials and methods}

Preparation of monoclonal antibody. The anti-human seminal plasma protein hybridoma cell strain was provided by The First Affiliated Hospital of Zhengzhou University (Zhengzhou, China), which was used for secretion of the monoclonal antibody of anti-human seminal plasma protein. Plasmids of Escherichia coli JM109 (pUC19) were preserved in the laboratory (First Affiliated Hospital of Zhengzhou University). The restriction endonuclease, Taq DNA polymerase, dNTP, buffer, purification system of Wizard ${ }^{\mathrm{TM}} \mathrm{Plus}$ Minipreps and the expression vector of the Glutathione S-transferase fusion protein (pGEX-4T-1) were purchased from Promega Corporation (Fitchburg, WI, USA); The rapid DNA ligation kit was purchased from Boehringer Mannheim GmbH (Basel, Switzerland); The Advantage ${ }^{\mathrm{TM}} \mathrm{PCR}-\mathrm{Pure}$ kit was purchased from Clontech Co. (Tokyo, Japan).

The key instruments used in the present study included a polymerase chain reaction (PCR) DNA Amplifier, a pipettor and refrigerated centrifuge (Eppendorf, Hamburg, Germany), a GFL-7601 incubator (GFL Company, Lower Saxony, Germany), a water bath (Shanghai Senxin Biological Technology Co., Ltd., Shanghai, China), a horizontal electrophoresis apparatus, a gel imaging system (Liyyi Company, Beijing, China) and a $-80^{\circ} \mathrm{C}$ ultra cold storage freezer (Haier, Beijing, China).

Extraction and reverse transcription of total cellular (c)DNA. The extraction of total cDNA was performed was performed using the DNA one step extraction method, as described previously (8). The total RNA was extracted from the cells and dissolved in $30 \mu \mathrm{l}$ RNase-free water, and reverse transcription was performed using $5 \mu \mathrm{l}$, according to the manufacturer's instructions (Promega Corporation).

Amplification of variable region genes of the monoclonal antibody. The genes of VH and VL were amplified using $672 \mathrm{ng} /$ $\mu 1$ template cDNA. The primer sequences were as follows: VH, reverse 5'-AGGT (CG) (AC) A (AG) CTGCAG (CG) AGTC (AT) GG-3' (degenerate primer) and forward 5'-TGA GGAAAC GGTGACCGTGGTCCCTTGGCCCCAG-3'; VL, reverse 5'-GTGAATTCGACATCGTGATGACCCAGT CTCC-3' and forward 5'-CAGTCGACTAACGTTTGATCT CCAGCTTGG TCCC-3' (Sangon Biotech, Shanghai, China). The PCR reaction system $(20 \mu \mathrm{l})$ included cDNA $(1 \mu \mathrm{l})$, dNTP $(0.4 \mu \mathrm{l}$ ), upstream and downstream primers (each $0.3 \mu \mathrm{l}$ ), Taq DNA polymerase $(0.2 \mu \mathrm{l})$, buffer $(2 \mu \mathrm{l})$ and sterilized water $(15.8 \mu 1)$. The reaction conditions included a denaturing stage for $60 \mathrm{sec}$ at $94^{\circ} \mathrm{C}$, annealing for $90 \mathrm{sec}$ at $55^{\circ} \mathrm{C}$, extension for $120 \mathrm{sec}$ at $72^{\circ} \mathrm{C}$, for 30 cycles. Following extracting $5 \mu \mathrm{l}$ amplicon from each of the $\mathrm{VH}$ and $\mathrm{VL}$ samples to perform the lipid sugar electrophoresis detection, the remaining amplicon was recycled by Advantage ${ }^{\mathrm{TM}}$ PCR-Pure kit according to the manufacturer's instructions and then was sequenced by the Shenggong Bioengineering Limited Company (Shanghai, China).

Modeling. The corresponding amino acid sequence was acquired by translating $\mathrm{VH}$ and $\mathrm{VL}$ gene sequences in $\gamma$-Sm-ScFv. The amino acid sequence of the protein

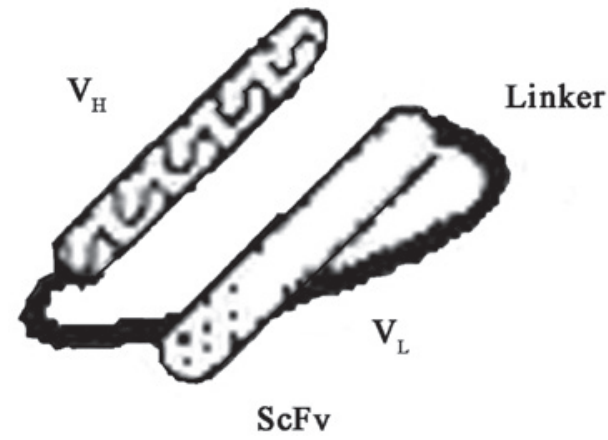

Figure 1. Structure of the $\mathrm{ScFv}$ antibody. $\mathrm{VH}$ indicates the heavy chain and VL indicates the light chain. ScFv, single chain variable fragment.

determined the advanced structure. Firstly, tertiary-structure modeling of VH and VL were performed using the homologous modeling method. The amino acid sequences of $\mathrm{VH}$ and VL were sent to SWISS-MODEL for modeling (http:// www.swissmodel.expasy.org/), and the acquired data file of the protein data bank structure was treated as the original structural file.

A total $\mathrm{ScFv}$ sequence can be combined by adding linker amino acid sequences and connecting them between $\mathrm{VH}$ and VL. Among which, (Gly4Ser)n was selected for the linker sequence. (Gly4Ser) refers to five short amino acid peptides, which are composed of four glycines and one serine. This oligopeptide is a repeated unit in the linker peptide, which is used for ligation of the VL and VH segments. It has relatively high flexibility or bending capacity. The small steric hindrance is useful for the interaction of the VL and VH segments, and arrangement of the correct conformation, as well as improving the stability of antibodies. Such structure is not easily recognized or degraded by proteases, which contributes to the stability of antibodies within the body. The combinatorial $\mathrm{ScFv}-\mathrm{n}$ sequence was also used in the modeling, and the data of the overall three-dimensional structure and that of VHn and VLn were obtained.

Structural contrast. To investigate the effect of the length of the linker peptide on the structure of VH and VL, the structural similarity of VH and VL in (Gly4Ser)n was calculated at different values of $n$. In addition, a space spherical shell hierarchical matching algorithm, based on spherical coordinates and described by Zhang and Chen (9), was used to calculate the level of similarity. In this algorithm, the protein was treated as the spherome, and the euclidean coordinates of each atom in the protein were transformed into spherical coordinates. According to the radius, the protein was then divided into several layers of spherical shells, and the same number of same atoms in each layer were collected. On the basis of predetermined weights, the number of atoms were determined, thus, obtaining the final value of atoms in each layer. Each layer of atomic values of $\mathrm{VH}-(\mathrm{Gly} 4 \mathrm{Ser}) \mathrm{n}$ or VL-(Gly4Ser)n and VH (or VL) were stacked into vector a and vector $b$, respectively. The included angle cosine function was treated as a similarity function. The formula used (1), the algorithm of which was achieved using MATLAB 2012a software (The MathWorks, Inc., Natick, MA, USA) was as follows: 
Table I. Results of similarity using a space spherical shell hierarchical matching algorithm.

\begin{tabular}{lcccc}
\hline & \multicolumn{3}{c}{ Similarity } & \\
\cline { 2 - 4 } Chain & Layer 2 & Layer 3 & Layer 4 & Average \\
\hline VH0 & 0.8910 & 0.7653 & 0.6183 & 0.7582 \\
VH1 & 0.9033 & 0.6320 & 0.7680 & 0.7678 \\
VH2 & 0.9029 & 0.7931 & 0.5616 & 0.7525 \\
VH3 & 0.9076 & 0.8376 & 0.5866 & $\mathbf{0 . 7 7 7 3}$ \\
VH4 & 0.9073 & 0.8198 & 0.5866 & 0.7712 \\
VH5 & 0.9083 & 0.8299 & 0.5825 & 0.7736 \\
VH6 & 0.9085 & 0.8299 & 0.5825 & $\mathbf{0 . 7 7 3 6}$ \\
VH7 & 0.9085 & 0.8258 & 0.5861 & 0.7735 \\
VH8 & 0.9085 & 0.8258 & 0.5861 & 0.7735 \\
VL0 & 0.9482 & 0.9723 & 0.8021 & 0.9075 \\
VL1 & 0.9515 & 0.9726 & 0.7992 & 0.9078 \\
VL2 & 0.9515 & 0.9729 & 0.7992 & 0.9079 \\
VL3 & 0.9515 & 0.9734 & 0.7997 & $\mathbf{0 . 9 0 8 2}$ \\
VL4 & 0.9515 & 0.9726 & 0.7992 & 0.9078 \\
VL5 & 0.9515 & 0.9729 & 0.7992 & 0.9079 \\
VL6 & 0.9515 & 0.9734 & 0.8018 & $\mathbf{0 . 9 0 8 9}$ \\
VL7 & 0.9507 & 0.9734 & 0.7997 & 0.9079 \\
VL8 & 0.9507 & 0.9734 & 0.7997 & 0.9079 \\
\hline
\end{tabular}

Numbers in bold indicate a high degree of similarity. VH, heavy chain; VL, light chain.

$$
\cos (a, b)=\frac{\sum_{i=1}^{n} a_{i} b_{i}}{\sqrt{\sum_{i=1}^{n} a_{i}^{2} \sum_{i=1}^{n} a_{i}^{2}}}
$$

Stability analysis. The stability of $\mathrm{ScFv}$ is presented as the fore and aft distance or the diffusion radius. The fore and aft distance refers to the distance from the fore $\alpha$-carbon atom to the aft $\alpha$-carbon atom. Using MATLAB, the fore and aft distances of the original structure of $\mathrm{VH}$ and $\mathrm{VL}$ were calculated, respectively, adding the fore and aft distances of VHn and VLn when $n=0-9$. In addition, their largest diffusion radius was calculated.

\section{Results}

The optical density $(\mathrm{OD})_{260} / \mathrm{OD}_{280}$ value of the overall extracted mRNA was 2.1, according and its concentration was $1,279 \mathrm{ng} / \mu \mathrm{l}$, which met the requirements of the experiment. The concentration of cDNA transcribed by reverse transcription was $672 \mathrm{ng} / \mu \mathrm{l}$. The cDNA was diluted at 1:50 and was used to amplify of the $\mathrm{VH}$ and the VL of the seminal plasma protein's single-chain antibody using PCR. The results of the electrophoresis are shown in Fig. 2.

The results of the $\mathrm{VH}$ and $\mathrm{VL}$ modeling in $\mathrm{ScFv}$ using the homologous modeling method are shown in Figs. 3 and 4. Generally, the length of the linker peptide of ScFv was between

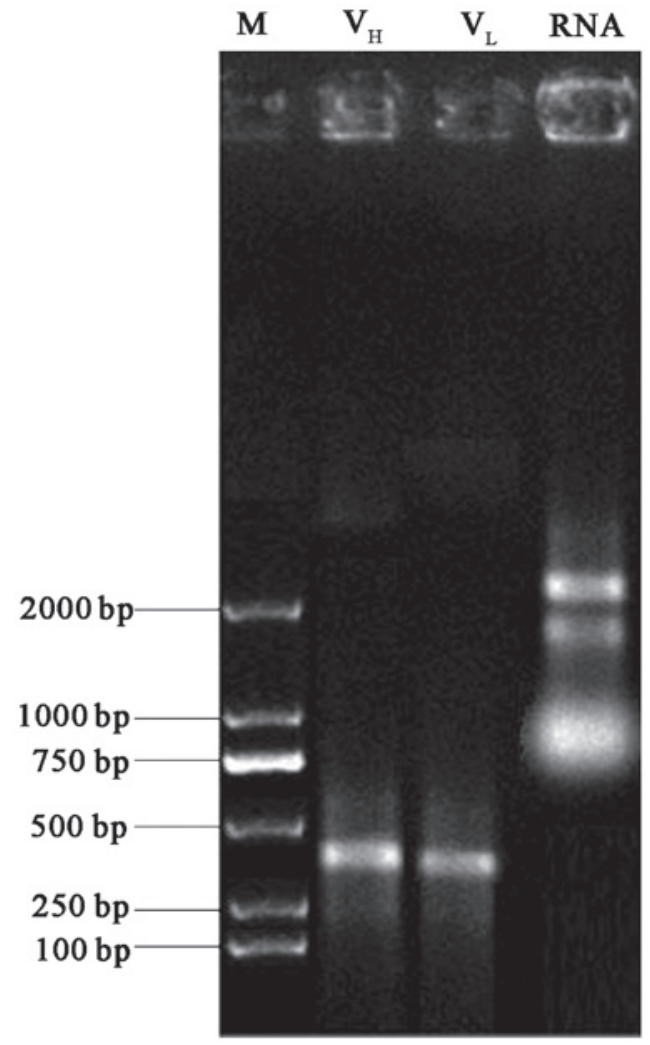

Figure 2. Gel electrophoresis, performed with separate separation of $\mathrm{VH}$ and VL. VH, heavy chain; VL, light chain; M, marker.
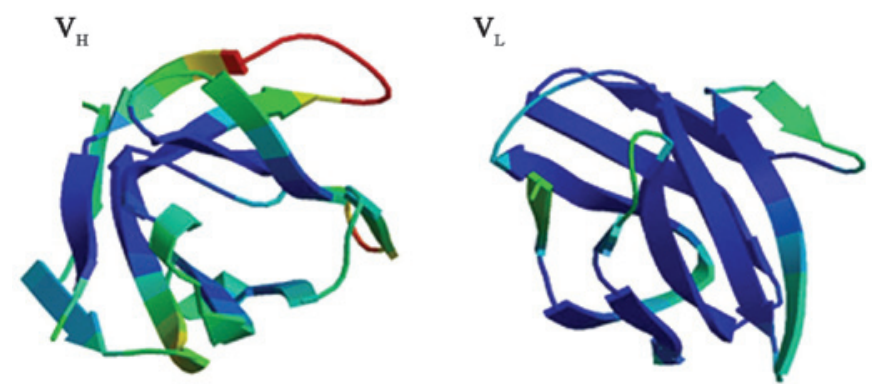

Figure 3. Single original antibody Swiss-models when linker $n=0$ and $n=3$. $\mathrm{VH}$, heavy chain; VL, light chain.

10 and 40 amino acids long. The longer the length of the linker peptide, the less stable the structure of the ScFv. During the process of modeling, when $n=9$ (linker peptide length of 45 amino acids), modeling failed due to the low homology of the searched templates. Therefore, three-dimensional models were obtained when the n-value was between 0 and 8 , and the results of the modeling are shown in Fig. 4. Each model had corresponding data for the VHn and VLn structure and data for the overall $\mathrm{ScFv}$-n structure.

From the figures described above, there was a clear difference in the structural models between VH/VL alone and $\mathrm{VH} / \mathrm{VL}$ when the linker was added, and the structure of the linker changed markedly as its length changed. Subsequently, the protein similarity algorithm, based on delamination of the spherical coordinates, was used to provide a contrast in the $\mathrm{VH}$ and VL similarity prior to and following addition of the linker. The results of the algorithm are shown in Table I. 
Table II. Changes of the fore and aft distance and radius of VH, VL and linker prior to and following addition of the linker.

\begin{tabular}{|c|c|c|c|c|}
\hline Chain & $\begin{array}{c}\text { Fore and } \\
\text { aft distance }\end{array}$ & Radius & $\begin{array}{l}\text { Length of } \\
\text { linker }\end{array}$ & $\begin{array}{c}\text { Fore and aft } \\
\text { distance of linker }\end{array}$ \\
\hline VHO & 32.9989 & 23.2127 & & \\
\hline VH1 & 42.3431 & 35.2561 & & \\
\hline VH2 & 37.1628 & 26.1484 & & \\
\hline VH3 & 41.6467 & 25.6413 & & \\
\hline VH4 & 38.8902 & 27.3090 & & \\
\hline VH5 & 40.5697 & 28.6009 & & \\
\hline VH6 & 40.3791 & 28.3365 & & \\
\hline VH7 & 40.8331 & 28.1269 & & \\
\hline VH8 & 40.8454 & 28.1225 & & \\
\hline VH & 39.9601 & 25.2179 & & \\
\hline VL0 & 22.3826 & 25.8404 & 0 & - \\
\hline VL1 & 19.0852 & 25.8461 & 5 & 14.021 \\
\hline VL2 & 19.0833 & 25.8452 & 10 & 13.5976 \\
\hline VL3 & 19.0739 & 25.8434 & 15 & 17.3828 \\
\hline VL4 & 19.0852 & 25.846 & 20 & 25.2548 \\
\hline VL5 & 19.0740 & 25.8428 & 25 & 16.7704 \\
\hline VL6 & 19.0659 & 25.8402 & 30 & 16.5636 \\
\hline VL7 & 19.0846 & 25.8444 & 35 & 17.7174 \\
\hline VL8 & 19.0745 & 25.8442 & 40 & 18.0869 \\
\hline VL & 36.7271 & 22.6299 & - & - \\
\hline
\end{tabular}

VH, heavy chain; VL, light chain.
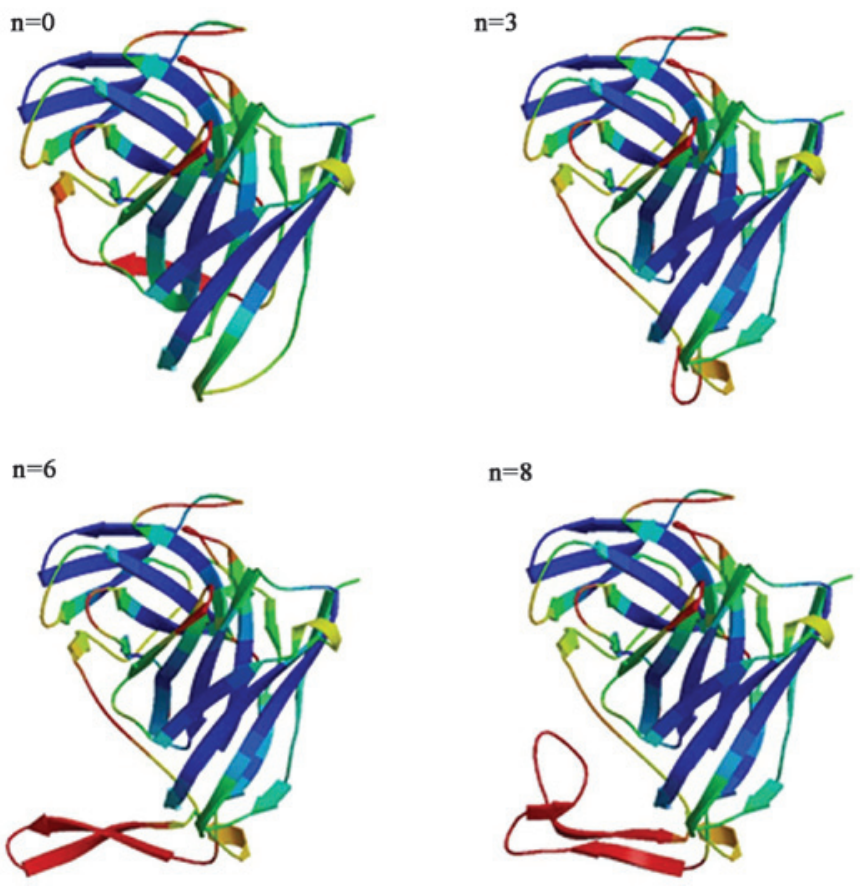

Figure 4. Single antibody models with linker lengths of $n=6$ and $n=8$.

Following several trials, the similarity data was relatively accurate and stable when the protein was divided into three layers. To guarantee the accuracy of the results, the protein was divided into 2, 3 and 4 layers. Subsequently, the mean similarity value of the data in each group was calculated and treated as the final result. As shown in Table I, when $n=3$ and $\mathrm{n}=6$, the similarity data obtained was close to the original data of VH and VL. The similarity has indicated the effect of the linker peptide's length on the variable region structure to the $\mathrm{ScF}_{\mathrm{v}}$, and the larger the value of the similarity, the lower the effect, which represented that the effect on the biological activity of a single-chain may be smaller.

Following the addition of the linker, the stability of $\mathrm{VH}$ and VL was analyzed again, based on the fore and aft distance and the diffusion radius. The results are shown in Table II.

As shown in Table II, when $n=6$, the fore and aft distance (40.3791) of the VH was the closest to that of VH (39.9601); when $n=3$, in which the diffusion radius (25.6413) was also closest to the original data (25.2179). By contrast, in the VL, the fore and aft distance following addition of the linker reduced to almost half that of the original data, however, the diffusion radius increased. In addition, when $n \leq 3$, the fore and aft distance of the linker increased as the length of the peptide increased, whereas, when $n>3$, the fore and aft distance decreased due to folding of the lengthened peptide chain. The changes in the fore and aft distance also affected VH and VL. The position comparison in the space coordinates system of the original VH, VL and the $\alpha$ carbon atoms of the ScFv-n structure was established by MATLAB 2012a, as is shown in Figs. 5 and 6, which suggested the fore and aft distance change of $\mathrm{VH}$ and VL was as expected. This indicated that after adding linker peptides of different lengths, the change in the whole molecular radius was small. 

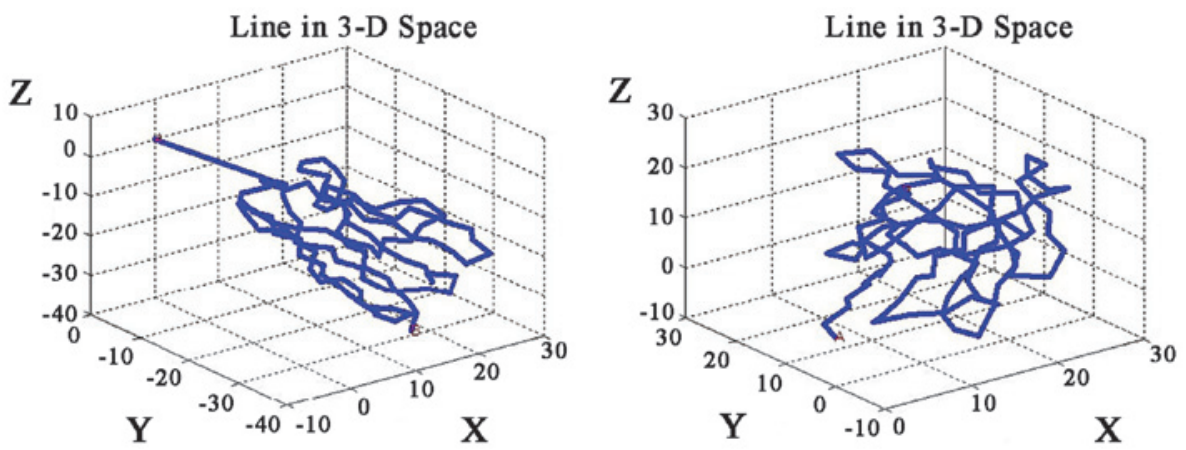

Figure 5. Original path of $\alpha$ carbon atom of a heavy chain and light chain.
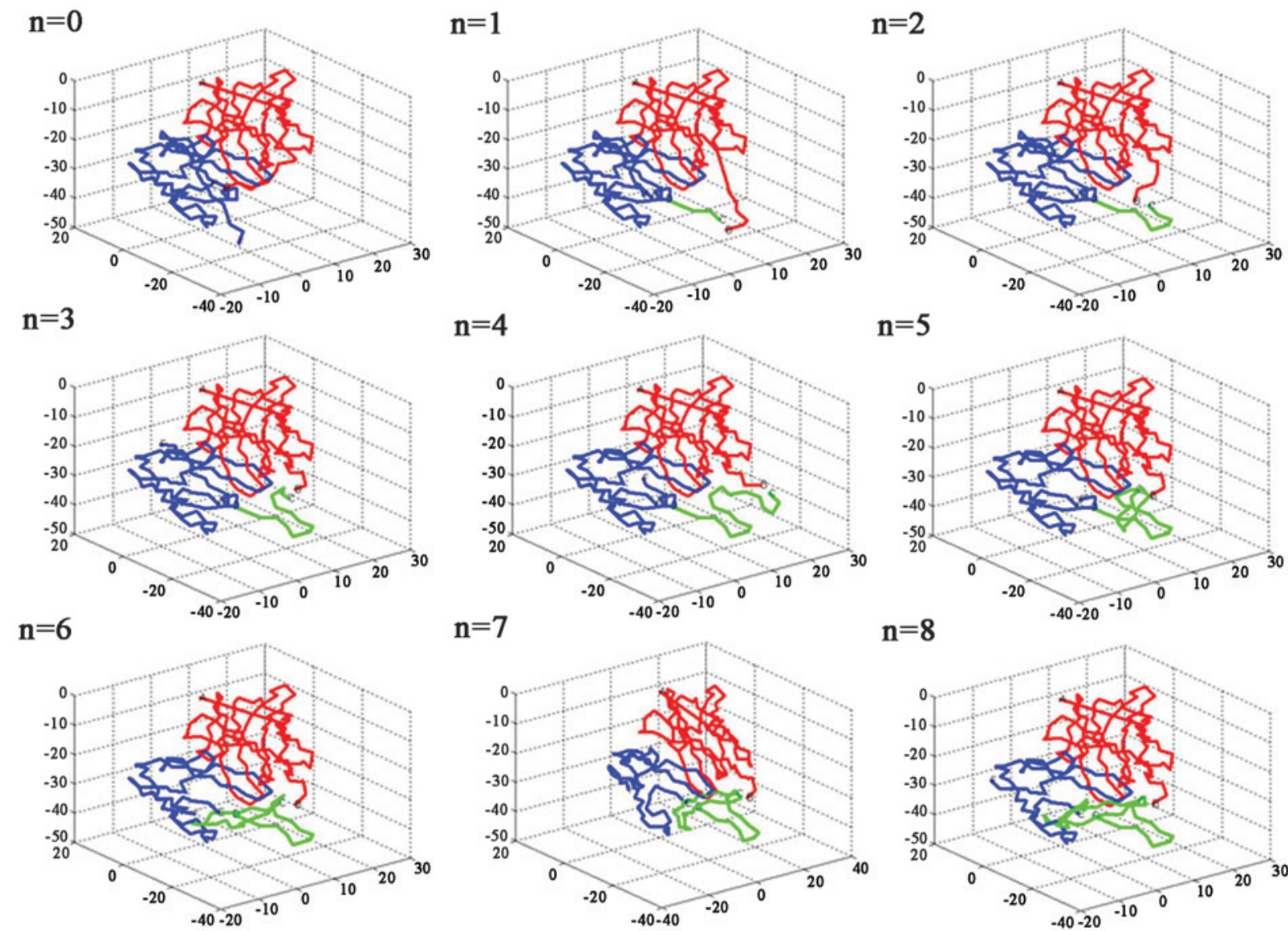

Figure 6. Path of $\alpha$ carbon atoms in a single-chain variable fragment when $n=1,2,3,4,5,6,7$ and 8 . Red indicates the heavy chain, blue indicates the light chain and green indicates the linker.

\section{Discussion}

From the comparative investigation performed in the present study, it was concluded that, in the spatial distribution of analogous proteins, the three-dimensional structure of proteins was more conserved than the primary sequence. The prediction of target protein spatial conformation is more reliable when performed in analogous proteins (10), and there have been several previous successes in predicting the structure of antibodies $(10,11)$. Successful modeling of the 6B4 antithrombotic antibody by Fontayne et al (11) laid a foundation for the investigation of its antithrombotic activity and antigenicity. Modeling of the three-dimensional structure of a protein can assist in the modification of the original antibodies on the basis of understanding its spatial structure and physiological functions, and it is significant for antibody engineering. ScFv molecule is a small molecule with weak immunogenicity and a rapid response time. It has no cumulative action in the kidney and its sharpness and clarity in tumor imaging is high, which enable it to be used as a vector and combined with medicine, isotopes and toxins (12). Single-chain antibody constructs belong to minimolecular antibodies. They are constructed by $\mathrm{VH}, \mathrm{VL}$ and linker peptides, and are the smallest functional fragments of antibodies in retention of the antigen-binding site, which has significant theoretical and applicable value in the diagnosis and treatment of cancer. 
In the present study, a series of methods, including modeling and bioinformatics, were used to examine and compare the structural changes in $\mathrm{ScFv}$ when the length of the linker peptide was altered. (Gly4Ser)n, as a linker peptide, is often selected in single-chain antibodies, among which 'Gly' represents glycine, 'Ser' represents serine and ' $n$ ' indicates the number of (Gly4Ser). The results of the present study revealed that, following addition of the linker, the similarity data for $\mathrm{VH}$ and VL was closest to that of the original data when $\mathrm{n}=3$ and $\mathrm{n}=6$, which indicated that $\mathrm{ScFv}-3$ and $\mathrm{ScFv}-6$ were closest to the original structure, as was their biological activity. In terms of stability, the fore and aft distance, and the diffusion radius of the $\mathrm{VH}$ and $\mathrm{VL}$ altered to different degrees following addition of the linker. Notably, when $n=6$, the fore and aft distance of the $\mathrm{VH}$ was closest to the original data, while that of the VL decreased by half. Compared with the original radius, that of $\mathrm{VH}$ and $\mathrm{VL}$ increased. When $\mathrm{n}=3$, however, the diffusion radius of the $\mathrm{VH}$ was the closest to the original data. The majority of the results indicated the the significance of the effect of linkers $n=3$ and $n=6$ on VH and VL.

Despite the construction of $\mathrm{ScFv}$ or a single-strand bispecific antibody, the linker peptide cannot affect the folding of the structure of an antibody or its biological activities (13). Several studies have investigated the effect of the length of linkers on the activities of ScFv (14). Kikuchi et al (15) constructed an anti-CD47 bivalent single-chain antibody, MABLsc(Fv), by way of covalence, using linkers of 15 amino acids (Gly4Ser); Yan et al (16) found that a linker length of 15 amino acids was more favorable for the folding of antibodies, guaranteeing the affinity of the bivalent single-chain antibodies. Goel et al (17) successfully constructed bivalent single-chain antibodies with immune activity using a longer linker of 25 amino acids. The present study confirmed that when $n=3$ (linker with 25 amino acids), the two variable regions in the $\mathrm{ScFv}$ were closest to the original structure. The results also demonstrated that, when $n=6$, the effect of the linker on the structure of $\mathrm{ScFv}$ was smaller. Considering that the present study used a bioinformatics approach, and that there may be a certain level of error in molecular modeling, the results of the present study require confirmation using biological experiments. Whether a linker length of 30 amino acids is suitable for the favorable expression of biological activity in a single-chain antibody also requires further investigation.

The effects of structural changes of linker peptides in single-chain antibodies on the whole antibody molecules were examined using mathematical modeling and bioinformatics methods, providing a basis for further investigation of the preparation of single-chain antibodies.

\section{Acknowledgements}

The authors would like to thank their colleagues within the department for their general support. This study was supported by the National Natural Science Foundation of China (grant no. 60971110), the Science and Technology Corporation Project of Henan Province (grant no. 122106000042) and the Open Science and Technology Corporation Project of Henan Province in 2013 (grant no. 132106000064).

\section{References}

1. Peng P, Gong YM, Bao PP, et al: Estimates and prediction of prostate cancer incidence, mortality and prevalence in China in 2008. Zhonghua Liu Xing Bing Xue Za Zhi 33: 1056-1059, 2012 (In Chinese).

2. Arai Y, Yoshiki T, Okada K and Yoshida O: Multiple marker evaluation in prostatic cancer using prostatic specific antigen, gamma-seminoprotein and prostatic acid phosphatase. Urol Int 44: 135-139, 1989.

3. Hao XK and Liang GD: Diagnosis of prostate cancer by radioimmunoimaging of 131I-antihuman seminal plasma protein. Natl Med J China 73: 655-657, 1993.

4. Hao XK, Wu GJ, Bai YJ, Yao LB, Zhang YH and Gao L: Construction and expression of single-chain Fv Genes of Anti-human seminal plasma protein. Chin J Urol 29: 30-32, 1999.

5. Fei JX, Ren J and Miao JY: Research on the Construction, expression and clinical application of single-chain antibody. Shaanxi Med J 29: 30-32, 2000.

6. Wu HG and Yan DF: Construction and expression of the variable region of single-chain antibody. J Taizhou Teach Coll 17: 55-59, 1995.

7. Yang LJ, Hou YC, Bai YJ, Yao LB and Su CZ: Analysis of primary structure and modeling of spatial structure of single-chain variable region of antibody against human gastric cancer. Shijie Huaren Xiaohua Zazhi 16: 2333-2336, 2008.

8. Chomczynski P and Sacchi N: Single-step method of RNA isolation by acid guanidinium thiocyanate-pheno-chloro form extraction. Anal Biochem 162: 156-159, 1987.

9. Zhang JH and Chen ZT: Analyzing influence on the conformation of single-chain antibody with the differential length of linkers. Afr J Microbiol Res 5: 5737-5744, 2011.

10. Thompson AJ, Price KL, Reeves DC, Chan SL, Chau PL and Lummis SC: Locating an antagonist in the 5-HT3 receptor binding site using modeling and radioligand binding. J Biol Chem 280: 20476-20482, 2005.

11. Fontayne A, Vanhoorelbeke K, Pareyn I, Van Rompaey I, Meiring M, Lamprecht S, Roodt J, Desmet J and Deckmyn H: Rational humanization of the powerful antithrombotic anti-GPIbalpha antibody: 6B4. Thromb Haemost 96: 671-684, 2006.

12. Colcher D, Bird R, Roselli M, Hardman KD, Johnson S, Pope S, Dodd SW, Pantoliano MW, Milenic DE and Schlom J: In vivo tumor targeting of a recombinant single-chain antigen-binding protein. J Natl Cancer Inst 82: 1191-1197, 1990.

13. Ming Fang, Xin Jiang and Zhi Yang: Influences of connecting peptide between the chain on the biological activity of ScBsAb. Chin Sci Bull 48: 1912-1918, 2003.

14. Zhang J, Shang Z and Zhang X: The optimization design of single bivalent connecting peptide antibodies. Math Med Biol 21: 602-605, 2008.

15. Kikuchi Y, Suzuki Y and Tamiya N: The source of oxygen in the reaction catalysed by collagen lysyl hydroxylase. Biochen J 213: 507-512, 1983.

16. Yan D, Fang J and Song J: Construction and Expression of Bivalent Single-chain Antibodies with Different Linker Sequence Against Human Colorectal Carcinoma. J Cell Biol 29: 272-276, 2007.

17. Goel A, Colcher D, Baranowska-Kortylewicz J, et al: Genetically engineered tetravalent single-chain $\mathrm{Fv}$ of the pan carcinoma monoclonal antibody CC49: improved bio distribution and potential for therapeutic application. Cancer Res 60: 6964-6971, 2000. 